\title{
Multiorgan Disorder Suggests Mitochondrial Disease
}

\author{
Josef Finsterer, MD, PhD ${ }^{1 *}$; Sinda Zarrouk-Mahjoub, $\mathrm{PhD}^{2}$ \\ ${ }_{1}^{1} 2^{\text {nd }}$ Medical Department with Cardiology and Intensive Care Medicine, \\ Krankenanstalt Rudolfstiftung, Vienna, Austria \\ ${ }^{2}$ University of Tunis El Manar and Genomics Platform, Pasteur Institute of Tunis, Tunis, Tunisia
}

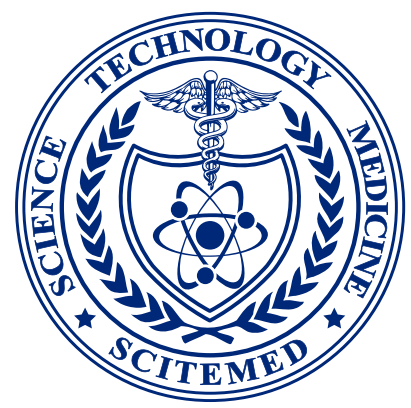

$\mathrm{n}$ a recent article, Reda et al. reported about a 41 -year-old patient with a classical mitochondrial multiorgan disorder syndrome (MIMODS), manifesting as stroke-like episodes (SLEs), epilepsy, ataxia, migraine, cognitive impairment, brain atrophy, hypoacusis, short stature, hypertrophic cardiomyopathy, heart failure, renal impairment, anemia, gout, and hyperlipidemia [1]. We have the following comments and concerns.

Diagnosing a mitochondrial disease can be facilitated by lactate-stress-testing [2]. Lactate-stress-testing represents a reliable screening test for suspected mitochondrial disorders based on the serum lactate elevation upon exercise on a bicycle ergometer with constant low effort below the lactate threshold. What were the results of this test?

Cerebrospinal-fluid lactate is frequently elevated in patients with a mitochondrial disorder. Cerebrospinal-fluid lactate can be determined either by direct measurement of lactate in the cerebrospinal fluid after a spinal tap or by indirectly measuring the lactate concentration by proton magnetic resonance spectroscopy. However, cerebrospinal-fluid lactate was obviously not determined since it remained unreported.

Stroke-like lesions typically present with regional hyperperfusion on three-dimensional pulsed arterial spin labeling. Did the stroke-like lesions in the presented patient also manifest with hyperperfusion on perfusion magnetic resonance imaging studies or scintigraphy?

SLEs typically manifest with weakness, aphasia, hemianopsia, epileptic activity on electroencephalogram (EEG), or seizures. Were the EEGs

\section{Article Information}

*Correspondence: Josef Finsterer, MD, PhD

Krankenanstalt Rudolfstiftung, Postfach 20, 1180, Vienna, Austria.

Email: fifigs1@yahoo.de

Dr. Finsterer and Dr. Zarrouk-Mahjoub contributed to this paper equally.

Received:Jul. 03, 2018; Accepted:Jul. 12, 2018; Published:Aug. 07, 2018

DOI:_10.24983/scitemed.cmt.2018.00073

Copyright (c) 2018 The Author(s). This is an open-access article distributed under the terms of the Creative Commons Attribution 4.0 International License (CC-BY).

Funding: None

Conflict of Interest: None with epileptogenic activity recorded and were the seizures a manifestation of recurrent SLEs or independent of them [3]?

In mitochondrial encephalomyopathy, lactic acidosis, and stroke-like episodes (MELAS) patients carrying the m.3243A>G variant, the mutation usually occurs in a heteroplasmic distribution. Unfortunately, no heteroplasmy rates of the m.3243A>G variant were provided from any tissue. Since the patient underwent autopsy, it would be interesting to know the heteroplasmy rates in clinically affected and non-affected organs, particularly if the post-mortem heteroplasmy rate was the highest in the brain, as one might expect.

The family history suggests maternal transmission but genetic studies in relatives are lacking. Heteroplasmic mitochondrial DNA (mtDNA) point mutations may occur spontaneously in $25 \%$ of the cases [4], why it is more likely that the presumed mtDNA mutation was inherited.

Nitric oxide precursors (L-arginine, L-citrulline) are particularly beneficial intravenously for acute SLEs [5]. Were they administered during SLEs and were they beneficial?

Overall, this interesting case could profit from the provision of a more profound imaging and genetic work-up and from genetic studies in maternal relatives. We should also be informed about the results of prospective investigations for a MIMODS, frequently manifesting as a multisystem disease.

\section{Keywords}

Genotype; mitochondrial; mtDNA; muscle biopsy; myopathy; phenotype.

\section{References}

1. Reda HM, Copen WA, Karaa A, Oakley DH. Case 13-2017. A 41-yearold man with hearing loss, seizures, weakness, and cognitive decline. N Eng/J Med 2017;376(17):1668-1678.

2. Finsterer J, Milvay E. Stress lactate in mitochondrial myopathy under constant, unadjusted workload. Eur J Neurol 2004;11(12):811-816.

3. Fryer RH, Bain JM, De Vivo DC. Mitochondrial encephalomyopathy lactic acidosis and stroke-like episodes (MELAS): a case report and critical reappraisal of treatment options. Pediatr Neurol 2016;56:59-61.

4. Poulton J, Finsterer J, Yu-Wai-Man P. Genetic counselling for maternally inherited mitochondrial disorders. Mol Diagn Ther 2017;21(4):419-429.

5. Kitamura $M$, Yatsuga $S, A b e T$, et al. L-Arginine intervention at hyper-acute phase protects the prolonged MRI abnormality in MELAS. J Neurol 2016;263(8):1666-1668. 